\title{
Mortality of Adult Critically Ill Subjects With Cancer
}

\author{
Regis Goulart Rosa MD PhD, Tulio Frederico Tonietto MD, Bruno Achutti Duso MD, \\ Juçara Gasparetto Maccari MD PhD, Roselaine Pinheiro de Oliveira MD PhD, \\ William Rutzen MD, Laura Madeira MD, Aline Ascoli MD, Rachel Hessler MD, \\ Paola Morandi MD, Ricardo Viegas Cremonese MD, Felippe Leopoldo Dexheimer Neto MD PhD, \\ Luciana Tagliari MD, Patrícia de Campos Balzano MD, José Hervê Diel Barth MD, and \\ Cassiano Teixeira MD PhD
}

\begin{abstract}
BACKGROUND: Cancer patients may require intensive care support for postoperative care, complications associated with underlying malignancy, or toxicities related to cancer therapy. The higher mortality rates found in this population than in the population of ICU patients without cancer may be attributable to confounding due to a higher prevalence of multiple organic dysfunctions at ICU admission in patients with malignancy; however, data regarding this hypothesis are scarce. Accordingly, we performed the present study to compare the crude and propensity score-matched mortality rates between adult subjects with and without cancer admitted to a mixed medicalsurgical ICU. METHODS: We conducted a retrospective analysis of a comprehensive longitudinal ICU database in a tertiary referral hospital in Southern Brazil. All adult subjects who were admitted to the ICU from January 2008 to December 2014 were evaluated. Crude and propensity score-matched all-cause 30-d mortality rates of critically ill subjects with cancer were compared with those of critically ill subjects without cancer. RESULTS: A total of 4,221 subjects were evaluated. The survival analysis revealed that the crude mortality rate was higher among subjects with cancer than among subjects without cancer $(18.7 \%$ vs $10.2 \%, P<.001)$. However, after matching by propensity score, the 30-d mortality rates of subjects with and without cancer were similar (18.5\% vs $15.2 \%, P=.17)$. CONCLUSIONS: The present study failed to show an association between malignancy and all-cause 30-d mortality rate in adult subjects admitted to a mixed medical-surgical ICU. The propensity score-matched analysis showed no evidence of excessive mortality due to cancer diagnosis. Key words: critical illness; critical care; cancer; mortality; prognosis. [Respir Care 2017;62(5):615-622. (C) 2017 Daedalus Enterprises]
\end{abstract}

\section{Introduction}

Patients with cancer are living longer nowadays. ${ }^{1}$ With advancements in diagnosis and treatment, even for ad-

\footnotetext{
Drs Rosa, Tonietto, Maccari, de Oliveira, Rutzen, Madeira, Balzano, Barth, Ascoli, and Teixeira are affiliated with the ICU; Drs Duso and Hessler are affiliated with the Department of Internal Medicine; Drs Morandi and Cremonese are affiliated with the Department of Palliative Care; and Dr Tagliari is affiliated with the Rapid Response Team, Hospital Moinhos de Vento, Porto Alegre, Brazil. Dr Oliveira is also affiliated with the ICU, Santa Casa de Misericordia Hospital, Porto Alegre, Brazil. Dr Teixeira is also affiliated with the Department of Internal Medicine, UFCSPA Medical School, Porto Alegre, Brazil. Dr Dexheimer is affiliated with the ICU, Ernesto Dornelles Hospital, Porto Alegre, Brazil.
}

vanced cases, malignant neoplasms are being controlled and long-term remission is being achieved. Similarly, the admission of oncologic patients to ICUs has become increasingly common, given the chronic disease status that cancer has reached. Patients with malignant neoplasms may require admission to the ICU for various reasons, usually respiratory failure, renal failure requiring renal replacement therapy, severe sepsis or septic shock, drug tox-

\footnotetext{
Correspondence: Cassiano Teixeira MD PhD, Moinhos de Vento Hospital, Ramiro Barcelos, 910/605, Porto Alegre-RS 90640-010, Brazil. E-mail: cassiano.rush@gmail.com.
}

DOI: $10.4187 /$ respcare. 05210 


\section{Mortality of CRitically Ill Subjects With Cancer}

icities related to medical treatment, and postoperative care. Currently, it is estimated that these patients account for $15-18 \%$ of all ICU admissions, and this rate is expected to increase with increases in long-term survival in this population. ${ }^{2,3}$

Early studies have shown that the mortality rates for subjects with cancer were substantially higher than those for subjects without cancer, suggesting that the presence of malignancy could be classified as a gloomy prognostic factor in the critical care scenario. ${ }^{4,5}$ This belief has long been used to support refusing ICU admission or withdrawing intensive support early in cancer patients, especially for those with advanced disease and in the context of reduced ICU bed availability. However, more recently published literature indicates that the mortality rate for critically ill subjects with cancer is decreasing ${ }^{6}$ and that the mere diagnosis of malignancy may no longer represent a strong predictor of ICU mortality, with mortality rates similar to those of subjects without cancer or even lower than those of subjects with some specific diseases, such as advanced heart failure. ${ }^{2,3,7}$ These studies showed that ICU mortality is more closely related to the number and severity of organ dysfunctions during ICU stay than to the cancer diagnosis per se, suggesting the existence of confounding in the relationship between cancer diagnosis and ICU mortality. To clarify the role of cancer mortality in critically ill subjects, we performed this study with the objective of comparing the crude and propensity scorematched mortality rates between subjects with and without cancer admitted to a single medical-surgical ICU in Southern Brazil.

\section{Methods}

\section{Study Design, Subjects, and Setting}

A retrospective analysis of a comprehensive longitudinal ICU database was conducted at a single tertiary center. The present study followed all ICU subjects $>18$ y old who were consecutively admitted to the 31-bed mixed medical-surgical ICU of the Hospital Moinhos de Vento in Porto Alegre, Brazil from January 2008 to December 2014. Patients who were receiving palliative treatment only or had an ICU stay of $<24 \mathrm{~h}$ were excluded. Subjects were not allowed to re-enter the study after their first ICU admission.

The ICU evaluated in the present study had the same policy for ICU admission of cancer subjects during the study period: first, all admissions of cancer subjects were determined jointly by the oncology and intensive care teams; second, subjects with poor performance status and no cancer treatment options were not considered for ICU admission; third, a broad policy of admission to the ICU was encouraged to avoid inappropriate ICU refusal.

\section{QUICK LOOK}

\section{Current knowledge}

The higher mortality rates found for cancer patients than for ICU patients without cancer may be attributable to confounding due to a higher prevalence of multiple organic dysfunctions at ICU admission in those patients with malignancy.

\section{What this paper contributes to our knowledge}

In the crude model analyses, cancer subjects had a higher rate of organ dysfunction and a greater need for intensive support (ventilatory, hemodynamic, and renal support). After one-to-one propensity score-matched analysis to address selection bias, we found no evidence of higher mortality in critically ill subjects due to cancer diagnosis. Therefore, the higher mortality of subjects with cancer admitted to the ICU is due to greater development of multi-organ dysfunction and not the cancer itself.

\section{Sample Size Calculation}

To detect a $10 \%$ difference in all-cause $30-\mathrm{d}$ mortality between subjects with and without cancer in the ICU with a power of $90 \%$ and a type- 1 error rate of 0.05 by using a Pearson chi-square test, we calculated that 379 pairs of propensity score-matched subjects would be required. This calculation considered an all-cause 30-d mortality rate of $27 \%$ among cancer ICU subjects. ${ }^{3}$ To avoid reduction of power due to follow-up losses, the present study protocol determined evaluation of at least 419 pairs of propensity score-matched subjects.

\section{Definitions}

Subjects admitted to the ICU were classified as cancer subjects depending on the diagnosis of solid or hematologic neoplasia before ICU admission. Subjects with a past history of cancer and with complete remission for $>5 \mathrm{y}$ were placed in the non-cancer group.

The type of ICU admission was classified into a dichotomous variable: admission due to a medical condition or surgery. Subjects were classified as admitted due to a surgery if they were admitted to the ICU immediately after a surgery (early postoperative period). Subjects admitted in the late postoperative period due to complications that did not require surgical interventions were classified as medical patients. We made this classification, because the outcomes of patients admitted to the ICU in the late postop- 


\section{Mortality of CRitically Ill Subjects With Cancer}

erative period are more closely related to medical than to early postoperative surgical patients. ${ }^{8}$

Infection was defined as clinically suspected infection (pneumonia, bloodstream infections [including infective endocarditis], intravascular catheter-related infection, intra-abdominal infections, urinary tract infections, surgical wound infections, skin and soft tissue infections, and central nervous system infections) according to the international sepsis forum consensus conference on the definitions of infection in the ICU. ${ }^{9}$ Severe sepsis and septic shock were defined according to the Surviving Sepsis Campaign guidelines. ${ }^{10}$ Subjects were defined as having ARDS if the arterial oxygen pressure to inspiratory oxygen fraction was $<300$, bilateral infiltrates were observed on the chest radiograph, and there was no clinical evidence of heart failure. ${ }^{11}$ The severity of the critical illness and the degree of organ failure were assessed within $24 \mathrm{~h}$ of ICU admission by the Acute Physiology and Chronic Health Evaluation II (APACHE II) score and the sequential organ failure assessment (SOFA) score, respectively. The ICU interventions performed within the first 24 and $72 \mathrm{~h}$ of ICU admission were evaluated by the simplified therapeutic intervention scoring system (TISS 28). APACHE II is a common scoring system used to grade the severity of illness in critically ill subjects. It generates a score ranging from 0 to 71 based on 12 physiologic variables, including age and variables describing the patient's underlying health: The higher the score, the greater the acute illness severity. ${ }^{12}$ The SOFA score is based on the extent of the patient's organic function determined by physiological parameters of the respiratory, neurologic, cardiovascular, hepatic, coagulation, and renal systems: The higher the score, the greater the number of organic dysfunctions. ${ }^{13}$ The TISS 28 score is based on interventions related to basic activities, including ventilator support, cardiovascular support, renal support, neurologic support, metabolic support, and specific interventions: The higher the score, the greater the number of interventions the patient is receiving. ${ }^{14}$

\section{Outcome, Follow-Up, and Data Management}

The primary outcome of this study was all-cause mortality $30 \mathrm{~d}$ after ICU admission. During the ICU stay, subjects were followed through interviews and medical record reviews by researchers who were not associated with the attending physician's team. For subjects who were discharged from the hospital in $<30 \mathrm{~d}$, follow-up telephone calls were made on the 30th day after ICU admission to determine whether they were still alive; if a subject was deceased at the time of the telephone call, the survival time was calculated based on the date of death reported by the family. Data were collected using preprinted case report forms. The data collection on ICU admission included demographic data; number of comorbidities; type of ICU admission (medical vs surgical); and diagnoses of infection, severe sepsis, septic shock, and ARDS. The data collected during the ICU stay included APACHE II, SOFA, and TISS 28 scores and specific interventions, such as mechanical ventilation, vasopressors, inotropes, renal replacement therapy, corticosteroids, neuromuscular blocking agents, and urgent surgery.

\section{Statistical Analysis}

Observational studies are often limited by an imbalance in both known and unknown confounders, which cause some cancer subjects to be more likely to develop unfavorable outcomes during their ICU stay than subjects without cancer. Therefore, we applied propensity score matching to balance baseline characteristics and reduce the probability of selection bias. ${ }^{15}$ The propensity score (probability of having cancer) was calculated using a multivariate logistic regression model in which the dependent variable was diagnosis of malignancy. Multi-collinearity was assessed according to the variance inflation factor of the multivariate model. ${ }^{16}$ The variance inflation factor estimates how much the variance of a coefficient is inflated because of the linear dependence of other predictors. Indicators of multi-collinearity include individual variance inflation factors of $>10$ or an average variance inflation factor of $>6$. Matching was performed with the use of a 1:1 matching protocol without replacement (nearest neighbor algorithm). ${ }^{17}$ Standardized differences were estimated for all of the baseline covariates before and after matching to assess prematch imbalance and postmatch balance. Standardized differences $\leq 10.0 \%$ for a given covariate indicated a small imbalance. Kaplan-Meier curves were used to calculate the time-dependent occurrence of death in both the unmatched and propensity score-matched cohorts; the log-rank test was used for comparisons between groups. The 30-d all-cause mortality rate was also evaluated with the Pearson chi-square test. A significance level of 0.05 was adopted for all statistical comparisons. The software used for the statistical analysis was STATA 12 (StataCorp, College Station, Texas).

\section{Ethics}

The Institutional Research Ethics Committee of the Hospital Moinhos de Vento, Porto Alegre, Brazil approved the study protocol and waived the requirement of informed consent.

\section{Results}

During the study period, 4,221 subjects (981 with cancer and 3,240 without cancer) were admitted to the ICU 


\section{Mortality of CRitically Ill Subjects With Cancer}

Table 1. Comparison of Variables Between Intensive Care Subjects With Cancer and Those Without Cancer

\begin{tabular}{|c|c|c|c|c|}
\hline \multirow{2}{*}{ Variables } & \multicolumn{2}{|c|}{$\begin{array}{l}\text { Entire Cohort } \\
(N=4,221)\end{array}$} & \multicolumn{2}{|c|}{$\begin{array}{l}\text { Propensity Score-Matched } \\
\text { Cohort }(n=842)\end{array}$} \\
\hline & $\begin{array}{l}\text { Cancer group } \\
(n=981)\end{array}$ & $\begin{array}{l}\text { Non-Cancer Group } \\
\quad(n=3,240)\end{array}$ & $\begin{array}{c}\text { Cancer Group } \\
(n=421)\end{array}$ & $\begin{array}{l}\text { Non-Cancer Group } \\
\quad(n=421)\end{array}$ \\
\hline Male sex, $n(\%)$ & $562(57.2)$ & $1,688(52.1)$ & $216(51.3)$ & $224(53.1)$ \\
\hline Age, mean \pm SD y & $69.2 \pm 14.2$ & $67.8 \pm 18.6$ & $69.0 \pm 14.5$ & $70.1 \pm 17.1$ \\
\hline Number of comorbidities, ${ }^{*}$ mean $\pm \mathrm{SD}$ & $3.0 \pm 0.9$ & $2.7 \pm 1.1$ & $2.9 \pm 0.9$ & $2.8 \pm 1.1$ \\
\hline Admission due to a medical condition, $n(\%)$ & $527(53.7)$ & $2,115(65.2)$ & $253(60.1)$ & $275(65.3)$ \\
\hline APACHE II, mean \pm SD & $17.5 \pm 8.1$ & $15.6 \pm 8.0$ & $16.8 \pm 7.8$ & $17.8 \pm 8.0$ \\
\hline SOFA at ICU admission, mean \pm SD & $3.6 \pm 3.4$ & $3.1 \pm 3.3$ & $3.9 \pm 3.3$ & $3.9 \pm 3.3$ \\
\hline Infection at ICU admission, $n(\%)$ & $407(41.4)$ & $1034(31.9)$ & $181(42.9)$ & $194(46.0)$ \\
\hline Severe sepsis at ICU admission, $n(\%)$ & $94(9.5)$ & $204(6.3)$ & $41(9.7)$ & $50(11.8)$ \\
\hline Septic shock at ICU admission, $n(\%)$ & $173(17.6)$ & $353(10.9)$ & $70(16.6)$ & $67(15.9)$ \\
\hline ARDS at ICU admission, $n(\%)$ & $41(1.2)$ & $19(1.9)$ & $9(2.1)$ & $8(1.9)$ \\
\hline Mechanical ventilation, $n(\%)$ & $451(45.9)$ & $1243(38.3)$ & $211(50.0)$ & $227(53.9)$ \\
\hline Vasopressor, $n(\%)$ & $442(45.0)$ & $1167(36.0)$ & $191(45.3)$ & $190(45.1)$ \\
\hline Inotrope, $n(\%)$ & $27(2.7)$ & $120(3.7)$ & $12(2.8)$ & $10(2.3)$ \\
\hline Corticosteroids, $n(\%)$ & $353(32.9)$ & $697(21.5)$ & $133(31.5)$ & $151(35.8)$ \\
\hline Neuromuscular blocking agent, $n(\%)$ & $31(3.1)$ & $80(2.4)$ & $14(3.3)$ & $16(3.8)$ \\
\hline Renal replacement therapy during ICU stay, $n(\%)$ & $135(13.7)$ & $384(11.8)$ & $64(15.2)$ & $65(15.4)$ \\
\hline Urgent surgery during ICU stay, $n(\%)$ & $57(5.8)$ & $194(5.9)$ & $29(6.8)$ & $24(5.7)$ \\
\hline TISS 28 after $24 \mathrm{~h}$, mean $\pm \mathrm{SD}$ & $22.0 \pm 8.5$ & $20.0 \pm 8.9$ & $22.4 \pm 8.0$ & $22.3 \pm 8.7$ \\
\hline TISS 28 after $72 \mathrm{~h}$, mean \pm SD & $18.4 \pm 9.4$ & $16.4 \pm 9.9$ & $18.3 \pm 8.8$ & $18.7 \pm 9.2$ \\
\hline \multicolumn{5}{|c|}{$\begin{array}{l}\text { * Heart failure, ischemic heart disease, cerebrovascular disease, diabetes mellitus, COPD, cirrhosis, HIV infection, chronic renal failure. } \\
\text { APACHE II = Acute Physiology and Chronic Health Evaluation II score }\end{array}$} \\
\hline
\end{tabular}

and were included in this study; $62.6 \%(n=2,642)$ were admitted due a medical condition, and $37.4 \%(n=1,579)$ were admitted due to a surgery. Among the subjects with cancer admitted to the ICU, 85.9\% (843 subjects) had solid neoplasms and $14.1 \%$ (138 subjects) had hematologic neoplasms. The rate of metastatic disease among subjects with solid neoplasms and the rate of relapsed disease among subjects with hematologic disease were $27.4 \%$ (231 subjects) and 53.6\% (74 subjects), respectively. The baseline clinical characteristics of all subjects evaluated in the present study are shown in Table 1. As a result of the non-randomized design of this study, the baseline characteristics of the subjects with cancer were different from those of the subjects without cancer in the unmatched cohort. The differences that were particularly important were sex; admission due to a medical condition; APACHE II score; infection, severe sepsis and septic shock at ICU admission; TISS 28 score at 24 and $72 \mathrm{~h}$ after ICU admission; and some specific ICU interventions, such as mechanical ventilation, administration of vasopressors and corticosteroids, and renal replacement therapy.

Several clinical factors were significantly associated with cancer diagnosis (Table 2): male sex, higher number of comorbidities, higher APACHE II score, infection at ICU admission, and requirement of corticosteroids. In contrast, admission due to a medical condition and the need for an inotrope and renal replacement therapy were negatively associated with the diagnosis of malignant neoplasm. The multivariate logistic regression model of factors associated with cancer diagnosis among critical care subjects was used to determine the propensity score. The analysis of the variance inflation factor showed no evidence of important multi-collinearity: the maximum individual variance inflation factor was 2.5 , and the average was 1.5 . Subjects with similar propensity scores were matched at a 1:1 ratio; as a result, 421 pairs of subjects were identified. In the cohort of propensity score-matched subjects, the standardized differences of all covariates between subjects with and without cancer were $<10 \%$, which suggested that the propensity score matching appropriately adjusted for the initial selection bias (Fig. 1). No follow-up losses occurred in the propensity score-matched cohort.

The all-cause $30-\mathrm{d}$ mortality rates in the overall study population were $12.2 \%$ (515 subjects) for the unmatched cohort and $16.8 \%$ (142 subjects) for the propensity scorematched cohort. The analysis of survival rates (Figs. 2 and 3) showed higher mortality rates for subjects with cancer than for those without cancer in the unmatched cohort (18.7\% vs $10.2 \%$, log-rank and chi-square $P<.001$ ); however, in the propensity score-matched cohort, subjects 
Table 2. Multivariate Logistic Regression Model of Baseline Factors Associated With Cancer Among Intensive Care Subjects (Propensity Score Model)

\begin{tabular}{|c|c|c|c|}
\hline Variable & Odds Ratio & $95 \% \mathrm{CI}$ & $P$ \\
\hline Male sex & 1.19 & $1.00-1.42$ & .042 \\
\hline Age, per year & 0.99 & $0.98-1.00$ & .09 \\
\hline Number of comorbidities* & 1.41 & $1.29-1.54$ & $<.001$ \\
\hline Admission due to a medical condition & 0.34 & $0.28-0.42$ & $<.001$ \\
\hline APACHE II, per point & 1.04 & $1.02-1.05$ & $<.001$ \\
\hline SOFA at ICU admission, per point & 0.96 & $0.93-1.00$ & .058 \\
\hline Infection at ICU admission & 1.31 & $1.05-1.62$ & .01 \\
\hline Severe sepsis at ICU admission & 1.18 & $0.86-1.60$ & .29 \\
\hline Septic shock at ICU admission & 1.07 & $0.80-1.44$ & .61 \\
\hline ARDS at ICU admission & 1.03 & $0.53-2.00$ & .91 \\
\hline Mechanical ventilation & 0.93 & $0.73-1.18$ & .57 \\
\hline Vasopressor & 1.00 & $0.79-1.27$ & .97 \\
\hline Inotrope & 0.57 & $0.33-0.99$ & .045 \\
\hline Corticosteroids & 1.47 & $1.19-1.82$ & $<.001$ \\
\hline Neuromuscular blocking agent & 0.93 & $0.56-1.55$ & .80 \\
\hline Renal replacement therapy during ICU stay & 0.70 & $0.53-0.92$ & .01 \\
\hline Urgent surgery during ICU stay & 0.68 & $0.47-0.97$ & .034 \\
\hline TISS 28 after $24 \mathrm{~h}$, per point & 1.00 & $0.98-1.01$ & .77 \\
\hline TISS 28 after $72 \mathrm{~h}$, per point & 1.00 & $0.99-1.01$ & .40 \\
\hline
\end{tabular}

* Heart failure, ischemic heart disease, cerebrovascular disease, diabetes mellitus, COPD, cirrhosis, HIV infection, chronic renal failure.

APACHE II = Acute Physiology and Chronic Health Evaluation II score

SOFA $=$ Sequential Organ Failure Assessment score

TISS $28=$ simplified therapeutic intervention scoring system

with cancer had the same all-cause 30 -d mortality as subjects without cancer $(18.5 \%$ vs $15.2 \%$, log-rank $P=.17$, chi-square $P=.19$ ). A post hoc power analysis showed that the present propensity score-matched cohort exhibited a power of $21 \%$ to detect a difference of $3.3 \%$ (18.5\% vs $15.2 \%$ ) for all-cause 30 -d mortality, considering a 2 -sided $\alpha$ of 0.05 . To reach a power of $80 \%$ to detect a $3.3 \%$ difference for mortality, 2,078 propensity score-matched pairs of subjects would be necessary.

\section{Discussion}

In the present study, the higher crude 30-d mortality found in ICU subjects with cancer than in those without cancer was due to confounding. In the crude model, subjects with cancer had a higher rate of organ dysfunction and a greater need for intensive support (eg, mechanical ventilation, vasopressor administration, and renal replacement therapy). Using a one-to-one propensity scorematched analysis to address selection bias, we found no evidence of higher mortality in critically ill subjects due to cancer diagnosis.

In the 1980s and 1990s, some studies highlighted the role of the intensivist refusing admission of patients with cancer to ICUs. Data published in this context showed unacceptable mortality rates of subjects with cancer despite intensive life support. ${ }^{5,18}$ At that time, the inclusion of some subjects with cancer, especially those with advanced disease, which underwent intensive therapeutic strategies, may have been disproportional, and those treatments may have been deleterious given the poor prognosis of that population. However, with the progressive advancements in the treatment of cancer and the supportive care provided to critically ill patients in recent decades, the long-term survival rates of both oncologic and critically ill patients have increased. This explains the current critical care background in which critically ill patients, both with and without cancer, can have comparable outcomes. Similar to our findings, several studies ${ }^{2,3,19,20}$ have shown that the presence of malignancy may no longer be an independent risk factor for death in the context of critical care. For instance, a large multi-center European study reported a mortality rate for subjects with solid cancer similar to that of ICU subjects without cancer. ${ }^{3}$ Moreover, the work of Tanvetyanon and Leighton ${ }^{7}$ showed that the in-hospital mortality of critically ill subjects with cancer was not higher than that of subjects with other comorbidities.

Our results should be interpreted with caution, especially in ICUs with a high prevalence of hematological neoplasms, given that our sample of critically ill subjects with hematological malignancy was $<15 \%$ of our total 


\section{Mortality of CRitically Ill Subjects With Cancer}

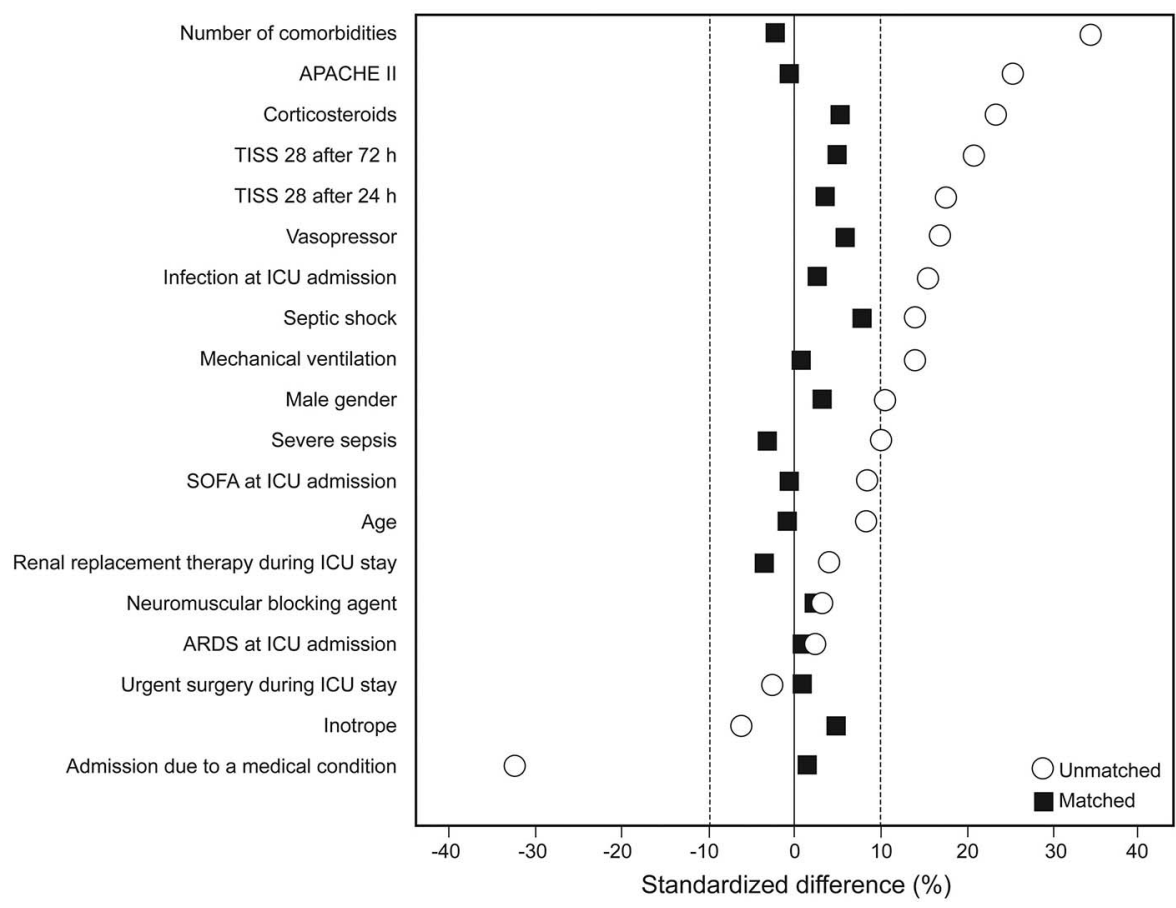

Fig. 1. Balance of covariates between critical care subjects with and without cancer and before and after propensity score matching. Note: After propensity score matching, 421 matched pairs were identified. The standardized differences are reported as percentages; a difference of $\leq 10.0 \%$ indicates a relatively small imbalance. APACHE II = acute physiology and chronic health evaluation II, TISS $28=$ therapeutic intervention scoring system, SOFA = sequential organ failure assessment.

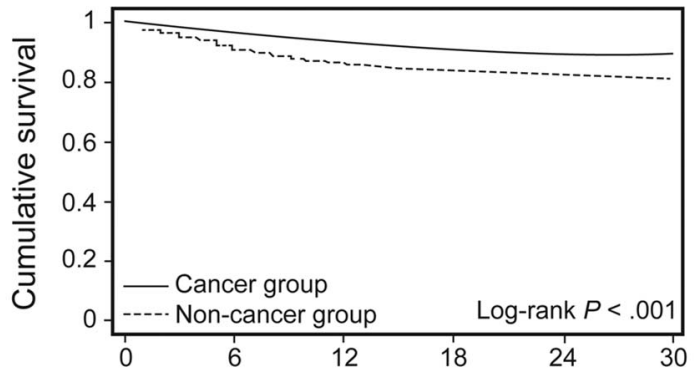

Subjects at risk $(n)$ Time (d)

Non-cancer group $3,240 \quad 3,128 \quad 3,032 \quad 2,963 \quad 2,929 \quad 2,909$

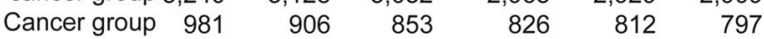

Fig. 2. Comparison of Kaplan-Meier survival curves between critical care subjects with and without cancer; crude analysis.

ICU cancer population. However, conflicting data also exist regarding the higher mortality risk among the subpopulation of cancer subjects with hematological malignancies. Although some large studies have found higher mortality rates for subjects diagnosed with hematological malignancies than for subjects diagnosed with solid neoplasms, ${ }^{3,21}$ some evidence suggests that the role of the type of malignancy in outcome determination in the setting of critical care illness is questionable. Accordingly, a prospective study by Soares et $\mathrm{al}^{2}$ with $>700,000$ subjects with cancer showed that mortality was predicted by severity of organ failures and performance status and not by

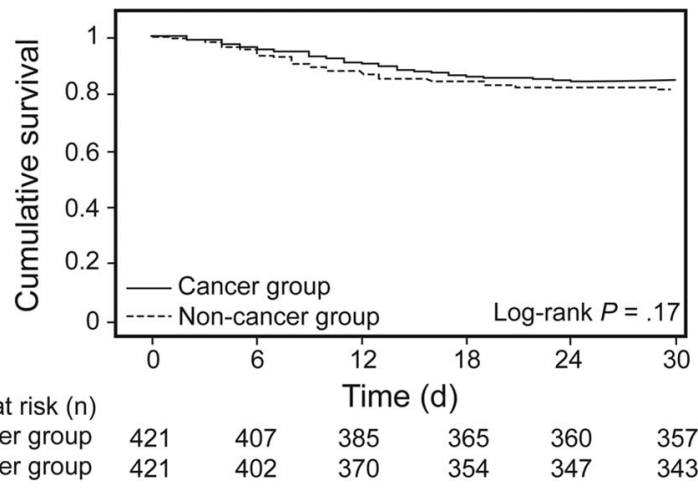

Fig. 3. Comparison of Kaplan-Meier survival curves between critical care subjects with and without cancer; propensity scorematched analysis.

cancer-related characteristics, such as the type of malignancy and neutropenia. Moreover, a study by Massion et $\mathrm{al}^{22}$ showed that the short-term prognosis of critically ill subjects with hematologic cancer is related to acute organ dysfunctions and pathogen aggressiveness; the severity of the underlying hematologic malignancy was not independently associated with mortality.

The strength of the present study includes the low risk of selection and confounding bias due to equivalence of important covariates between the 2 study groups in the propensity score-matched cohort. Previous studies evalu- 


\section{Mortality of CRitically Ill Subjects With Cancer}

ating cancer subjects admitted to ICUs used heterogeneous statistical analysis methods. ${ }^{2,3,5,15,20}$ Some statistical methods, such as standard multivariate regression, tend to inflate effects in observational studies, especially when the number of prognostic factors is high and when there is insufficient overlap of covariates between the 2 study arms. ${ }^{23}$ In contrast, the propensity score method creates a model that reflects the effects of risk factors on the exposure. Matching using a single summary variable that predicts the probability of being exposed as a function of the confounders creates a statistical model with fewer assumptions and may lead to improved estimates of exposure effects in some settings. ${ }^{24}$ Using a stringent and conservative matching analysis, we attempted to achieve causal inference of mortality.

Our study has limitations. Because of the one-to-one propensity score matching procedure with a narrow caliper, the sample size was reduced. Moreover, the mortality rate of our cohort was lower than expected, and the study was underpowered to detect differences $<10 \%$ of all-cause 30-d mortality between subjects with and without cancer. In addition, our study evaluated only short-term mortality of ICU subjects; this outcome may underestimate the true mortality of ICU patients, given that the mortality in the first months after ICU discharge is high, and ICU cancer patients may be particularly susceptible to this long-term outcome. ${ }^{25}$ Also, the lack of quality-of-life measurements among survivors represents a drawback: were survivors with and without cancer comparable with regard to quality of life? How did the ICU intervention impact dignity and distress among those cancer survivor subjects with reversible physiologic dysfunction but with a poor malignancy prognosis? Other important limitations of the present study were related to its single-center retrospective design; lack of control of important covariates associated with prognosis, such as delay between ICU referral and admission, type of malignancy, cancer progression, or previous allogeneic stem cell transplantation; and possible systematic errors related to observational studies, given that the propensity score can balance study groups with respect to measured covariates only, and we cannot be certain that we identified all potential confounding factors for mortality in the critical care scenario.

In conclusion, the diagnosis of cancer before ICU admission does not necessarily impact all-cause ICU mortality. ICU admission policies should be rationally enhanced to cover all potential beneficiaries of intensive care, taking into account the needs and perspectives of patients and their families. Currently, the best approach for critically ill cancer patients at ICU admission is to improve communication among the multidisciplinary oncology and critical care teams to offer early and complete vital support for those patients with a reasonable baseline prognosis (an ICU trial). ${ }^{26}$ After this full-code approach, there should be a predefined revaluation in 3-5 d of the number of recovered organ dysfunctions and response to therapies in course. Ideally, the participation in decisions of withholding and withdrawing life support should be discussed with a consultant palliative care specialist, considering patient and family preferences. ${ }^{27}$ After 2-3 weeks of stay in intensive care full support with ventilator, vasoactive drugs, and renal replacement therapy, a condition usually referred as chronic critical illness, ${ }^{28}$ the short- and long-term prognoses in the general ICU population are dismal and are probably much worse in the oncologic patient. One of the intensivist's attributions and competences to communicate the new prognosis to the oncologist and the family, helping them to deescalate treatment in the patient's best interest. Future research in the field of intensive care of cancer patients should explore the impact of ICU admission on overall long-term and disease-free survival as well as quality of life among ICU survivors with cancer.

\section{REFERENCES}

1. Siegel R, Ma J, Zou Z, Jemal A. Cancer statistics, 2014. CA Cancer J Clin 2014;64(1):9-29.

2. Soares M, Caruso P, Silva E, Teles JM, Lobo SM, Friedman G, et al. Characteristics and outcomes of subjects with cancer requiring admission to intensive care units: a prospective multicenter study. Crit Care Med 2010;38(1):9-15.

3. Taccone FS, Artigas AA, Sprung CL, Moreno R, Sakr Y, Vincent JL. Characteristics and outcomes of cancer subjects in European ICUs. Crit Care 2009;13(1):R15.

4. Darmon M, Azoulay E. Critical care management of cancer subjects: cause for optimism and need for objectivity. Curr Opin Oncol 2009; 21(4):318-326.

5. Price KJ, Thall PF, Kish SK, Shannon VR, Andersson BS. Prognostic indicators for blood and marrow transplant subjects admitted to an intensive care unit. Am J Respir Crit Care Med 1998;158(3):87684.

6. Pène F, Percheron S, Lemiale V, Viallon V, Claessens YE, Marqué, et al. Temporal changes in management and outcome of septic shock in subjects with malignancies in the intensive care unit. Crit Care Med 2008;36(3):690-696.

7. Tanvetyanon T, Leighton JC. Life-sustaining treatments in subjects who died of chronic congestive heart failure compared with metastatic cancer. Crit Care Med 2003;31(1):60-64.

8. Haas JS, Teixeira C, Cabral CR, Fleig AHD, Freitas APR, Treptow EC, et al. Factors influencing physical functional status in intensive care unit survivors two years after discharge. BMC Anesthesiol 2013; 13:11.

9. Calandra T, Cohen J. International Sepsis Forum Definition of Infection in the ICU Consensus Conference: the international sepsis forum consensus conference on definitions of infection in the intensive care unit. Crit Care Med 2005;33(7):1538-1548.

10. Dellinger RP, Levy MM, Rhodes A, Annane D, Gerlach H, Opal SM, et al. Surviving Sepsis Campaign: international guidelines for management of severe sepsis and septic shock: 2012. Crit Care Med 2013;41(2):580-637.

11. ARDS Definition Task Force, Ranieri VM, Rubenfeld GD, Thompson BT, Ferguson ND, Caldwell E, et al. Acute respiratory distress syndrome: the Berlin definition. JAMA 2012;307(23):2526-2533. 


\section{Mortality of CRitically Ill Subjects With Cancer}

12. Breslow MJ, Badawi. Severity scoring in the critically ill: part 1-interpretation and accuracy of outcome prediction scoring systems. Chest 2012;141(1):245-252.

13. Vincent JL, Moreno R, Takala J, Willatts S, De Mendonça A, Bruining H, et al. The SOFA (Sepsis-related Organ Failure Assessment) score to describe organ dysfunction/failure: on behalf of the Working Group on Sepsis-Related Problems of the European Society of Intensive Care Medicine. Intensive Care Med 1996;22(7):707-710.

14. Moreno R, Morais P. Validation of the simplified therapeutic intervention scoring system on an independent database. Intensive Care Med 1997;23(6):640-644.

15. Oakes JM, Church TR. Invited commentary: advancing propensity score methods in epidemiology. Am J Epidemiol 2007;165(10):11191121; discussion 1122-1123.

16. Midi H, Sarkar SK, Rana S. Collinearity diagnostics of binary logistic regression model. J Interdisciplinar Mathematics 2010;13(3): 253-267. doi: 10.1080/09720502.2010.10700699.

17. Austin PC. An introduction to propensity score methods for reducing the effects of confounding in observational studies. Multivariate Behav Res 2011;46(3):399-424.

18. Kress JP, Christenson J, Pohlman AS, Linkin DR, Hall JB. Outcomes of critically ill cancer Subjects in a university hospital setting. Am J Respir Crit Care Med 1999;160(6):1957-1961.

19. Thiéry G, Azoulay E, Darmon M, Ciroldi M, De Miranda S, Lévy V, et al. Outcome of cancer subjects considered for intensive care unit admission: a hospital-wide prospective study. J Clin Oncol 2005; 23(19):4406-4413.
20. Azoulay E, Mokart D, Pène F, Lambert J, Kouatchet A, Mayaux J, et al. Outcomes of critically ill subjects with hematologic malignancies: prospective multicenter data from France and Belgium: a groupe de research respiratoire en reanimation onco-hématologique study. J Clin Oncol 2013;31(22):2810-2818.

21. Oeyen SG, Benoit DD, Annemans L, Depuydt PO, Van Belle SJ, Troisi RI, et al. Long-term outcomes and quality of life in critically ill subjects with hematologic or solid malignancies: a single center study. Intensive Care Med 2013;39(5):889-898.

22. Massion PB, Dive AM, Doyen C, Bulpa P, Jamart J, Bosly A, Installé E, et al. Prognosis of hematologic malignancies does not predict intensive care mortality. Crit Care Med 2002;30(10):2260-2270.

23. Martens EP, Pestman WR, de Boer A, Belitser SV, Klungel OH. Systematic differences in the treatment effect estimates between propensity score methods and logistic regression. Int J Epidemiol 2008; 37(5):1142-1147.

24. Stuart EA. Matching methods for causal inference: a review and a look forward. Stat Sci 2010;25(1):1-21.

25. Brinkman S, de Jonge E, Abu-Hanna A, Arbous MS, de Lange DW, de Keizer NF. Mortality after hospital discharge in ICU subjects. Crit Care Med 2013;41(5):1229-1236.

26. Lecuyer L, Chevret S, Thiery G, Darmon M, Schlemmer B, Azoulay E. The ICU trial: a new admission policy for cancer subjects requiring mechanical ventilation. Crit Care Med 2007;35(3):808-814.

27. Aslakson RA, Curtis JR, Nelson JE. The changing role of palliative care in the ICU. Crit Care Med 2014;42(11):2418-2428.

28. Nelson JE, Cox CE, Hope AA, Carson SS. Chronic critical illness. Am J Respir Crit Care Med 2010;182(4):446-454. 